relationship quality, plays a critical role in disease, particularly for older wives.

\section{DYADIC PROFILES OF HEALTH BEHAVIORS AMONG KOREAN BABY BOOMER COUPLES}

B. Kim ${ }^{1}$, K. Kim, $\mathrm{PhD}^{2}$, J. Burr, $\mathrm{PhD}^{3}$, J. Kim, $\mathrm{PhD}^{4}$, G. $\operatorname{Han}^{5}$, 1. University of Massachusetts Boston, Boston, Massachusetts, United States, 2. University of Massachusetts Boston, Boston, MA, USA, 3. University of Massachusetts Boston, Boston, MA, USA, 4. Seoul National University, Seoul, Republic of Korea, 5. Seoul National University, Seoul, Republic of Korea

This study addresses the issue of whether health behaviors are concordant within couples. Specifically, we identified profiles of health behaviors among Korean baby boomer couples (born in 1955-1963; aged 53-61). We analyzed a sample of 1,096 middle-aged couples from the Korean Baby Boomer Panel Study (2014). Two health risk behaviors and four health preventive behaviors were included. Latent Class Analysis and multinomial regression were performed to describe and analyze underlying health behavior profiles. Four dyadic profiles of health behaviors were identified: a) high concordant-high preventive couples $(27 \%)$; b) high concordant-moderate preventive couples $(24 \%)$; c) moderate concordant-high preventive couples (14\%); and d) moderate concordant-low preventive couples $(35 \%)$. Couple's education, working status, health status, and marital quality were significantly different across profiles. Findings suggest that Korean baby boomer couples generally engage in concordant types and levels of health preventive behaviors, supporting the assortative mating and shared environment hypotheses.

\section{HIS AND HERS: HEALTH BENEFITS OF MARRIAGE IN THE CONTEXT OF MULTIMORBIDITY}

M. Franks ${ }^{1}$, P.A. Thomas, $\mathrm{PhD}^{2}$, J.L. Rector, $\mathrm{PhD}^{3}$, E.M. Friedman, $\mathrm{PhD}^{4}$, 1. Purdue University, West Lafayette, Indiana, United States, 2. Purdue University, West Lafayette, IN, USA, 3. Purdue University, West Lafayette, IN, USA, 4. Purdue University, West Lafayette, IN, USA;

Risk for chronic conditions increases with age, and many older adults manage multiple conditions (multimorbidity). Multimorbidity increases mortality risk, an association that is likely mediated through disability. Using data from the Survey of Midlife Development in the United States (MIDUS), we examined a mediated pathway linking multimorbidity to mortality through mobility limitations (Aim 1). Given established health benefits of marriage, we also examined potential moderating effects of marital status on this mediated pathway (Aim 2). In generalized structural equation models stratified by gender, being married was protective against mobility limitations among women with multimorbidity and against mortality among women with higher mobility limitations. Being married was more protective against mobility limitations and mortality among healthier men (i.e., those without multimorbidity or mobility limitations, respectively). Findings suggest that health benefits of marriage that protect functional ability and survival in the context of multimorbidity differ for men and women.
ORAL SEX FOR OLDER LOVERS: IMPLICATIONS ON RELATIONSHIP QUALITY AND MENTAL HEALTH H. Liu ${ }^{1}$, S. Shen, $\mathrm{MA}^{2}$, N. Hsieh, $\mathrm{PhD}^{3}$, 1. Michigan State University, East Lansing, Michigan, United States, 2. Department of Sociology, Michigan State University, East Lansing, MI, USA, 3. Department of Sociology, Michigan State University, East Lansing, MI, USA

We examine giving versus receiving oral sex, two processes that shape and are shaped by relationship quality, and how they predict well-being in late life. We analyze 884 heterosexual couples from the National Social Life, Health, and Aging Project (2010-11). Results from the Actor-Partner Interdependence Models suggest that older adults with better relationship quality gave oral sex to their partner more often than those with worse relationship quality; this association was stronger for men than women. While receiving oral sex was positively related to both men's and women's perceptions of relationship quality, women's relationship quality was more strongly linked to their partners' well-being than men's. Men's giving of oral sex was positively related to their own wellbeing through increasing their partner's perceived relationship quality. Findings suggest that oral sex plays an important but overlooked role in maintaining an active sexual life, quality relationship, and psychological vibrancy in late life.

\section{SESSION 1985 (SYMPOSIUM)}

\section{SOCIAL HEALTH AND DEMENTIA: INDIVIDUAL, INTERDISCIPLINARY AND INTERNATIONAL PERSPECTIVES}

Chair: A.M. Kolanowski, Penn State University, Kingston, Pennsylvania

Co-Chair: M. Roes, Sociology / Nursing Science, Witten, Nordrhein-Westfalen

Discussant: M. Downs, University of Bradford, Bradford, England

The definition of health should include a more dynamic focus on the ability of individuals to adapt and self-manage physical, mental and social well-being despite the presence of chronic conditions. In 2016 the European InterDem's Social Health Taskforce operationalized three dimensions of Social Health for dementia: (1) having the capacity to fulfil one's potential and obligations; (2) the ability to manage life with some degree of independence; and (3) participation in social activities. In this symposium we bring together presenters, both scientists and people living with dementia, who will share their personal experiences and data on the concept of Social Health in dementia. We begin with a brief overview of the concept from the European perspective. The first presentation is given by members of a stakeholder group of persons living with dementia who informed the U.S. 2017 National Research Summit on Dementia Care; they will expand on the moral imperative of facilitating the capacity to fulfil one's potential. The second presenter will report on findings from the NIA-funded COPE project that teaches family caregivers how to help their relatives with dementia manage life with greater independence by adapting the home environment to facilitate maximum function and safety. The final presenter will share data on the transferability and effectiveness of the Meeting Centres Support Programme, an intervention 